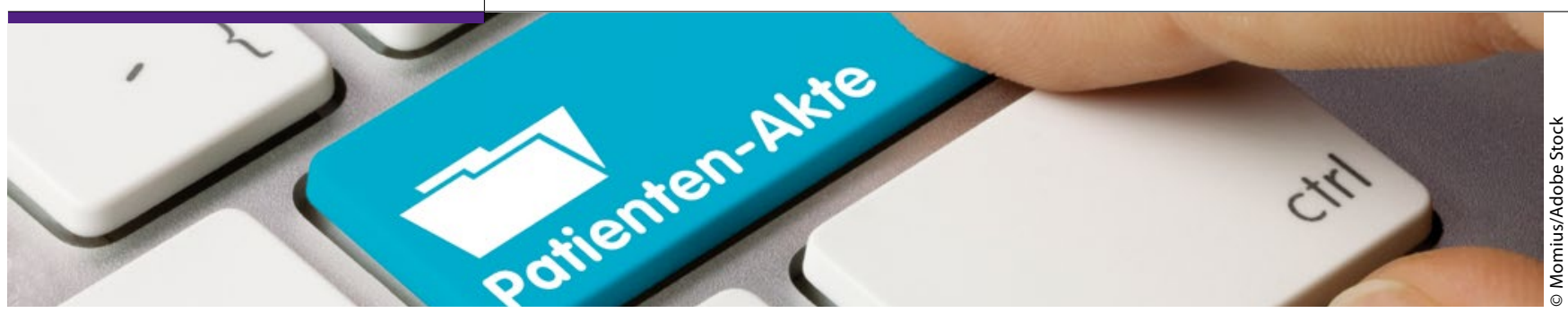

\title{
App-Daten für die Patientenakte
}

\author{
Eine direkte Datenschnittstelle von der Gesundheits-App in die Praxis- \\ EDV? Was nach Zukunftsmusik klingt, hat ein Praxissoftwareanbieter \\ nun umgesetzt. Eine Spam-Flut müssen die Ärzte aber trotzdem nicht \\ befürchten - wird versprochen.
}

m letzten Jahr tummelten sich 1,3 Millionen Gesundheits-Apps im GooglePlay-Store. Im Apple-iTune-Store waren es mit 1,2 Millionen nicht viel weniger (Quelle: statista.com). Nicht alle gelten als „echte“ Health-Apps, auch viele Fitness-Apps firmieren unter der Rubrik Gesundheit. Das Angebot wächst und weckt die Erwartung der Patienten, selbst Gesundheitsdaten zu erfassen und dem Arzt zur Verfügung zu stellen. Doch wie lassen sich die Daten ohne Mehraufwand strukturiert in die PraxisEDV übertragen? Und wer kanalisiert die Datenströme? Genau hier setzt der Arztsoftwareanbieter medatixx an. Mit seinem „medatixx-AppPoint" hat er eine Plattform geschaffen, auf der Ärzte und Patienten ausgewählte Health-Apps finden, die medizinische Inhalte sowie Datenschutzkonformität nachweisen. Das Entscheidende dabei: Die Apps werden über eine Schnittstelle mit den Praxisverwaltungssystemen des Unternehmens verbunden.

\section{Ein Datenkanal für alle Apps}

Technisch kommuniziert wird über den CDA(Clinical Document Architecture)Standard, der im medizinischen Bereich als Standard für den Datenaustausch zwischen unterschiedlichen Systemen gilt. Hier nicht wieder verschiedene $\mathrm{Da}-$ tenschnittstellen zu entwickeln, war für medatixx aus verschiedenen Gründen wichtig: Zum einen können sich die App-Entwickler so auf einen Standard einstellen. Damit können verschiedenste Health-Apps problemlos in den me-
datixx-AppPoint aufgenommen werden. Zum anderen können sich - theoretisch - so auch andere Praxis-EDV-Anbieter an den AppPoint andocken.

Die Daten werden von der App in die Praxissoftware immer verschlüsselt und eben strukturiert (statt in willkürlichem Format) transportiert. Als Datenmittler fungiert die medatixx-eigene Patientenservice-App „x-patient“. Über sie wird die sichere Verbindung zur Praxissoftware aufgebaut.

\section{QR-Code identifiziert Patient}

Es entscheidet der Arzt, welcher Patient ihm Daten übermitteln kann und aus welchen Apps heraus dies geschehen soll. Der Patient kann den Arzt also nicht mit Daten zuspammen. Um das PatientenSmartphone eindeutig der richtigen Karteikarte in der Praxissoftware zuordnen zu können, wird ein QR-Code generiert, den die Praxis an die Patienten vergibt, die sie mit der Praxissoftware verbinden will. Die Patienten scannen den Code über die x.patient-App mittels Smartphone-Kamera ein - und schon können die Datenströme fließen.

Der Softwareanbieter hat einen zusätzlichen Sicherheitspuffer eingebaut, um zu verhindern, dass zu viele Daten in der Praxis auflaufen. Alle Infos wandern erst einmal in eine Art Postfach, in die „Kommunikationssuite“. Der Arzt kann dann entscheiden, welche Daten in die elektronische Karteikarte eingespielt werden. Das ist auch hilfreich, weil Patienten über die App x.patient Wiederholungsrezepte bestellen oder direkt mit dem Praxisteam via Chatfunktion kommunizieren können.

Derzeit hat medatixx zwei HealthApps an seine Praxis-EDV-Systeme angebunden: „Preventicus Heartbeats“ für Patienten mit Herzrhythmusstörungen und die App „M-sense“ von Newsenselab für Migränepatienten, die unter anderem ein Migränetagebuch beinhaltet. Außerdem befindet sich mit "ARYA“, eine Smartphone-Anwendung zur Früherkennung und Unterstützung von Patienten mit Depressionen, eine weitere Health-App bereits in der Umsetzung.

Laut medatixx-Vertriebsleiter Michael Schober wird vor allem auf Indikationsgebiete gesetzt, die Ärzten einen Mehrwert in der Behandlung bringen. Das sei bei Anwendungen für chronisch kranke Patienten der Fall. „Wir stehen hier in engem Austausch mit den Ärzten.“

\section{Nur geprüfte Apps}

Die Bewertung der Apps nimmt medatixx nicht alleine vor. Hier setze man als Partner auf den Berliner Flying Health Incubator. Die Ideen-Schmiede unterstützt Start-ups dabei, digitale Diagnoseund Therapieanwendungen zur Marktreife zu bringen und zählt neben medatixx auch zum Beispiel die Barmer und die Sana Kliniken zu ihren Partnern. Mitbegründer ist zudem Dr. Markus Müschenich, der Kinderarzt und Gesundheitswissenschaftler ist auch Vorstandsmitglied im Bundesverband Internetmedizin und setzt sich seit Jahren für qualitative E-Health-Anwendungen ein. Kein Wunder also, dass die App Preventicus ein CE-zertifiziertes Medizinprodukt ist, das übrigens in der Premiumversion erlaubt, einen PDF-Report zu den Pulskurven des Patienten direkt aus der App heraus zu erzeugen und an die Praxissoftware zu übermitteln.

RebekkaHöhl 